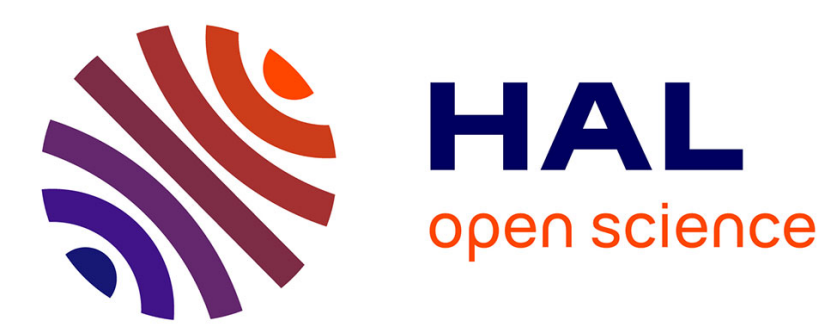

\title{
LES approach coupled with stochastic forcing of subgrid acceleration in a high-Reynolds-number channel flow
}

\author{
Rémi Zamansky, Ivana Vinkovic, Mikhael Gorokhovski
}

\section{To cite this version:}

Rémi Zamansky, Ivana Vinkovic, Mikhael Gorokhovski. LES approach coupled with stochastic forcing of subgrid acceleration in a high-Reynolds-number channel flow. Journal of Turbulence, 2010, 11, pp.118. 10.1080/14685248.2010.496787. hal-00566071

\section{HAL Id: hal-00566071 https://hal.science/hal-00566071}

Submitted on 28 Mar 2012

HAL is a multi-disciplinary open access archive for the deposit and dissemination of scientific research documents, whether they are published or not. The documents may come from teaching and research institutions in France or abroad, or from public or private research centers.
L'archive ouverte pluridisciplinaire $\mathbf{H A L}$, est destinée au dépôt et à la diffusion de documents scientifiques de niveau recherche, publiés ou non, émanant des établissements d'enseignement et de recherche français ou étrangers, des laboratoires publics ou privés. 


\title{
RESEARCH ARTICLE
}

\section{LES approach coupled with stochastic forcing of subgrid acceleration in a high Reynolds number channel flow}

\author{
Rémi Zamansky, Ivana Vinkovic and Mikhael Gorokhovski*
}

Laboratoire de Mécanique des Fluides et d'Acoustique, École Centrale de Lyon - CNRS Université Claude Bernard Lyon 1 - INSA Lyon, 36 avenue Guy-de-Collongue, F-69134

Écully Cedex, FRANCE

(v3.2 released February 2009)

\begin{abstract}
In this paper, the high Reynolds number channel flow is simulated by numerical approach at coarse resolution, in which the instantaneous acceleration is decomposed into filtered and subgrid parts, and then both components are modeled. The filtered acceleration is modeled in the framework of the LES approach. The model for the subgrid acceleration is based on two stochastic processes. The first is for its norm, and is based on statistical universalities in fragmentation under scaling symmetry, providing correlation of subgrid forcing across the channel. The second is for its orientation, and is based on the Brownian motion on a unit sphere in order to represent a stochastic relaxation towards full isotropy away from the wall. Two main parameters of the stochastic process include the Reynolds number based on the friction velocity, and the channel half-width. In order to assess the capability of the model proposed, the paper illustrates its application versus recent high Reynolds number DNS, including DNS performed in this paper.
\end{abstract}

Keywords: Turbulent channel flow, intermittency, LES, SGS model

\section{Introduction}

Application of large-eddy simulation (LES) in turbulent channel flow becomes too expensive when the Reynolds number is high, and the resolution of strong gradients in the near-wall region is needed. Starting from the earlier study of Moin and Kim [1], different modifications of this approach have been proven successful (Meneveau and Katz [2], Piomelli and Balaras [3], Sagaut [4], Pantano et al. [5], Spalart et al. [6]). Among them one is to incorporate direct simulation of subgridscale turbulence into LES techniques on the basis of estimation of the unresolved velocity field. Approaches proposed by Domaradzki and Adams [7] Park and Mahesh [8], and Westbury et al. [9] are focused on simulation of the residual-stress tensor. The model proposed by Schmidt et al. [10] is based on the LES/ODT (One Dimensional Turbulence) coupling [11, 12] and provides stochastically the unresolved velocity field as the wall is approached. The approach of Kemenov and Menon [13] is based on the simulation of a turbulent velocity field which is defined by superposition of large-scale and small-scale components. The latter is computed directly on subgrid-scales by a simplified 1D motion equation (in three directions). It is worth noting that the above reported subgrid-scale (SGS) models are invariant

*Corresponding author. Email: mikhael.gorokhovski@ec-lyon.fr

ISSN: $1468-5248$ (online only)

(C) 2010 Taylor \& Francis

DOI: $10.1080 / 14685240 Y Y x x x x x x x$

http://www.informaworld.com 
to the Reynolds number. 'I'hereby, in these models, the intermittency effects are discarded on subgrid-scales.

In the approach proposed by Sabel'nikov et al. in [14], the key element of the SGS model is acceleration in the residual flow. This acceleration has been modeled stochastically in the case of three-dimensional box turbulence, with statistical properties depending on the Reynolds number. The general idea of this approach, referred to as LES-SSAM (stochastic subgrid acceleration model), consists of the following. The total instantaneous acceleration

$$
\begin{aligned}
& \frac{d u_{i}}{d t}=-\frac{1}{\rho} \frac{\partial p}{\partial x_{i}}+\nu \frac{\partial^{2} u_{i}}{\partial x_{k} \partial x_{k}} \\
& \frac{\partial u_{k}}{\partial x_{k}}=0
\end{aligned}
$$

is composed of two contributions:

$$
\frac{d u_{i}}{d t}=\overline{\left(\frac{d u_{i}}{d t}\right)}+\left(\frac{d u_{i}}{d t}\right)^{\prime}
$$

where $\frac{d u_{i}}{d t}=\frac{\partial u_{i}}{\partial t}+u_{k} \frac{\partial u_{i}}{\partial x_{k}}$ The first component is the filtered total acceleration:

$$
\begin{aligned}
\overline{\left(\frac{d u_{i}}{d t}\right)} & =\frac{d \bar{u}_{i}}{d t}+\frac{\partial\left(\overline{u_{i} u_{k}}-\bar{u}_{i} \bar{u}_{k}\right)}{\partial x_{k}} \\
& =-\frac{1}{\rho} \frac{\partial \bar{p}}{\partial x_{i}}+\nu \frac{\partial^{2} \bar{u}_{i}}{\partial x_{k} \partial x_{k}} \\
\frac{\partial \bar{u}_{k}}{\partial x_{k}} & =0
\end{aligned}
$$

where the overbar denotes the filtering operation, $\bar{u}_{i}$ denotes the filtered velocity. The second component represents the total acceleration in the residual field:

$$
\begin{aligned}
\left(\frac{d u_{i}}{d t}\right)^{\prime} & =\frac{\partial u_{i}^{\prime}}{\partial t}+\frac{\partial\left(u_{k} u_{i}-\overline{u_{k} u_{i}}\right)}{\partial x_{k}} \\
& =-\frac{1}{\rho} \frac{\partial p^{\prime}}{\partial x_{i}}+\nu \frac{\partial^{2} u_{i}^{\prime}}{\partial x_{k} \partial x_{k}} \\
\frac{\partial u_{k}^{\prime}}{\partial x_{k}} & =0
\end{aligned}
$$

If both parts are modeled, their sum gives an approximation to (1).

The first assumption is to replace (3) by a classical LES-equation with a simple 
eddy-viscosity model

$$
\begin{aligned}
\overline{\left(\frac{d u_{i}}{d t}\right)_{\Delta}} & =\frac{d \bar{u}_{i}}{d t}-\frac{\partial}{\partial x_{k}}\left[\nu_{t}\left(\frac{\partial \bar{u}_{i}}{\partial x_{k}}+\frac{\partial \bar{u}_{i}}{\partial x_{k}}\right)\right] \\
& =-\frac{1}{\rho} \frac{\partial \bar{p}}{\partial x_{i}}+\frac{\partial}{\partial x_{k}}\left[\nu\left(\frac{\partial \bar{u}_{i}}{\partial x_{k}}+\frac{\partial \bar{u}_{i}}{\partial x_{k}}\right)\right] \\
\frac{\partial \bar{u}_{k}}{\partial x_{k}} & =0
\end{aligned}
$$

where $\nu_{t}$ denotes the eddy viscosity and subscript $\Delta$ is the length scale associated with the filter size.

The second assumption is to replace (4) by

$$
\begin{aligned}
\left(\frac{d u_{i}}{d t}\right)_{S}^{\prime} & =-\frac{1}{\rho} \frac{\partial p_{*}}{\partial x_{i}}+\hat{a}_{i}^{\prime} \\
\frac{\partial u_{k}^{\prime}}{\partial x_{k}} & =0
\end{aligned}
$$

where $p_{*}$ is the pressure providing the subgrid velocity model-field to be solenoidal, and $\hat{a}_{i}$ is attributed to the stochastic acceleration of the residual flow.

The third assumption is to replace the sum of (5) and (6) by the model-equation for a surrogate field, denoted hereafter by the symbol ^

$$
\frac{d \hat{u}_{i}}{d t}=-\frac{1}{\rho} \frac{\partial \hat{p}}{\partial x_{i}}+\frac{\partial}{\partial x_{k}}\left[\left(\nu+\nu_{t}\right)\left(\frac{\partial \hat{u}_{i}}{\partial x_{k}}+\frac{\partial \hat{u}_{k}}{\partial x_{i}}\right)\right]+\hat{a}_{i}^{\prime}
$$

In this equation, the new pressure field $\hat{p}$ is such that

$$
\frac{\partial \hat{u}_{k}}{\partial x_{k}}=0
$$

Equations (7) and (8) will be applied here to a high Reynolds number channel flow, with a new subgrid acceleration model for $\hat{a}_{i}^{\prime}$.

In high Reynolds number channel flows, the acceleration is a highly intermittent variable. It has been reported by direct numerical simulations (DNS) [15], that this intermittency is mostly related to quasi-coherent vortical structures in the wall-layer. A fluid particle, trapped in such a vortex, is subjected to an intense centripetal acceleration. It was revealed [15] that those fluid particles preserve the magnitude of their acceleration longer than its direction. This finding is consistent with the experimental studies [16-18] of a high Reynolds number "free" turbulence. Here, in wall-bounded turbulent flow, the intermittency effects are manifested by deep cross-channel interaction between energy containing eddies of very different length-scales [19]. Recent DNS studies identified mechanisms, which may be responsible for such turbulent correlations across the channel. In the near-wall region, there are upward ejections of vortical fluid, as a part of vortices-streaks regeneration cycle [20-25]. Above the buffer layer, where the Reynolds number dependent flow $[26,27]$ has intermittent streaky structure as well [28-30], the pressure fluctuations acting on the flow in the buffer zone and in the viscous sublayer, may 
also generate eruptions of small-scale wall structures towards outer How [31-33]. Besides, the unsteady bursting events, when the large outer-flow eddies appear closely to the wall, has been reported in [34]. Concerning LES of such flow, the significant role of the "under-resolved" momentum transfer across the channel was emphasized in [35].

The question raised in this study is as follows. Despite the huge number of very different turbulent structures, produced by different random realizations in the wall-bounded flow, and interacting across the scales, is it possible to match recent DNS statistics by a rather simple model in the framework of LES-SSAM approach. The purpose of this paper is to propose such a model with assessment of its capability by comparison with DNS. The subgrid acceleration model proposed here is based on the following assumptions : (i) there are two independent stochastic processes, one for the norm, and another for the orientation; (ii) both processes are correlated over the channel height; (iii) the stochastic process for the norm of acceleration is given by the statistical universalities of fragmentation under scaling symmetry; (iv) the stochastic process for the orientation relaxes towards isotropy in the channel center-line. Two main parameters of the stochastic process include the Reynolds number based on the friction velocity, and the channel half-width.

This paper is organized as follows. The stochastic model for the non-resolved acceleration will first be presented. The models for the norm and the orientation of the non-resolved acceleration will be described. Then, details about the performed numerical simulations will be given. Finally, the comparison between the classical LES, DNS and the LES-SSAM approach will be presented and discussed.

\section{Stochastic model for the non-resolved acceleration}

The stochastic behavior of the subgrid acceleration is prescribed to its norm and to its orientation. It is assumed that the orientation of subgrid acceleration is mostly controlled by orientation of vortex sheets and tubes, lying mostly in the streamwisespanwise plane near the wall. These structures become randomly distributed with increasing distance from the wall. As to the subgrid acceleration norm, it is constructed in terms of wall-parameters, following DNS observation [14], in which the significance of the solenoidal component of acceleration very near the wall was shown.

In this study, the suggestion is to introduce the stochastic processes for this norm with correlation across the channel. The subgrid forcing $\hat{a}^{\prime}$ in (7) is therefore given by two independent stochastic processes, one for its norm $|a|$ and another for its orientation $e_{i}$, i.e. :

$$
\hat{a}_{i}^{\prime}=|a| e_{i}
$$

This model will be different from the model proposed in [14], where authors introduced a model for the acceleration in homogeneous and isotropic turbulent flow given also by two independent stochastic processes, one for the norm of the acceleration, and another one for its orientation. In [14], the model for the acceleration norm has been derived from the lognormal model for the energy dissipation [36-40] (see also recent paper advocating the lognormal model, such as [41], and its application in [42]). Simultaneously its orientation was generated randomly in each 
time step of order of the Kolmogorov time scale.

\subsection{Model for the norm of the subgrid acceleration}

We represent the norm of the non-resolved acceleration by a typical velocity increment at the wall expressed as $\Delta \frac{u_{*}^{2}}{\nu}$ and multiplied by a frequency $f$, which is a random variable:

$$
|a|=\Delta \frac{u_{*}^{2}}{\nu} f
$$

Here $u_{*}$ is the friction velocity. High values of $f$ correspond to strong velocity gradients. Usually the stochastic process evolves with time. In order to introduce the cross-channel correlation in subgrid forcing by a stochastic process for $f$, we shall replace time by distance from the wall. Thus the random frequency $f$ will evolve stochastically from the wall to the outer flow. To this end, we introduce the non-dimensional evolution parameter $\tau$, which is defined as follows:

$$
\tau=-\ln \left(\frac{h-y}{h}\right)
$$

where $h$ is the channel half-width, and $y$ is the wall distance. It can be seen from (11) that when $y=0$ then $\tau=0$, and when $y \rightarrow h$ then $\tau \rightarrow \infty$. Hence $f$ evolves with $\tau$, increasing from zero to infinity, correspondingly to $y$ increasing from zero to the channel half-width.

The near-wall region is characterized by strong velocity gradients (high values of $f$ ), which decrease in mean towards the outer flow through a highly intermittent dynamics. We assumed that with increasing distance from the wall (increasing $\tau$ ) the frequency $f$ is changing by a random independent multiplier $\alpha(0<\alpha<1), f \rightarrow$ $\alpha f$. $\alpha$ is governed by distribution $q(\alpha), \int_{0}^{1} q(\alpha) d \alpha=1$. This multiplicative process, often referred to as fragmentation under scaling symmetry, requires knowledge of $q(\alpha)$, which is, in principle, unknown. However if relaxation of $f$ is fast, comparing to advancement from the wall to the outer region, the population balance equation, describing continuous evolution of distribution $G(f ; \tau)$ can be reduced exactly to the Fokker-Planck equation [43]:

$$
\frac{\partial G(f ; \tau)}{\partial \tau}=-\langle\ln \alpha\rangle \frac{\partial}{\partial f}(f G)+\frac{\left\langle\ln ^{2} \alpha\right\rangle}{2} \frac{\partial}{\partial f}\left(f \frac{\partial}{\partial f}(f G)\right)
$$

Here $G(f ; \tau)$ is the normalized distribution function, $\int_{0}^{\infty} G(f) d f=1$. It should be noted that only the first two logarithmic moments of $\alpha$, denoting here as $\left\langle\ln ^{k} \alpha\right\rangle=$ $\int_{0}^{1} q(\alpha) \ln ^{k} \alpha d \alpha, k=1,2$, appear in the evoluton of $G(f ; \tau)$ (equation 12 ) and $\langle\ln \alpha\rangle /\left\langle\ln ^{2} \alpha\right\rangle=\langle\ln f\rangle /\left\langle(\ln f-\langle\ln f\rangle)^{2}\right\rangle$, with $\langle\ln f\rangle=\int_{0}^{\infty} G(f) \ln f d f$.

The solution of (12) and its properties are described in [43]. It is shown that with increasing time (here with increasing of parameter $\tau, O<\tau<\infty$ instead of $O<t<\infty)$, the initial distribution $G(f ; \tau=0)$ goes first to the log-normal distribution:

$$
G(f ; \tau)=\frac{1}{F \sqrt{2 \pi\left\langle\ln ^{2} \alpha\right\rangle} \tau} \exp \left(-\frac{\left(\ln \frac{f}{F}\right)^{2}}{2\left\langle\ln ^{2} \alpha\right\rangle \tau}\right)\left(\frac{f}{F}\right)^{1-\frac{\langle\ln \alpha\rangle}{\left\langle\ln ^{2} \alpha\right\rangle}}
$$


where $F^{\prime}$ is the initial scale of frequency, which is introduced bellow. It is worth noting that originally, the lognormal distribution has been identified by Kolmogorov [44] in his discrete model of cascade breakage events. However the Kolmogorov's lognormality was derived in the context of the central limit theorem. The analytical solution (13) of the population balance equation, obtained without appealing to the central limit theorem, shows that by further increasing of $\tau$, the "log-normal" multiplier in eq. $13 \exp \left(-\left(\ln \frac{f}{F}\right)^{2} / 2\left\langle\ln ^{2} \alpha\right\rangle \tau\right)$ tends to unity, and the long-time limit distribution is determined by the power law with one universal parameter, $\langle\ln \alpha\rangle /\left\langle\ln ^{2} \alpha\right\rangle$. In our paper, instead of using those universal asymptotics, we use (12) in order to derive the stochastic equation. In the Itô interpretation, it reads :

$$
d f=\left(\langle\ln \alpha\rangle+\left\langle\ln ^{2} \alpha\right\rangle / 2\right) f d \tau+\sqrt{\left\langle\ln ^{2} \alpha\right\rangle / 2} f d W(\tau)
$$

where $d W(\tau)$ is a Wiener process $\left(\langle d W(\tau)\rangle=0\right.$ and $\left\langle d W(\tau)^{2}\right\rangle=2 d \tau$, here and below the brackets denote the ensemble average). Parameters are chosen in the following form:

$$
\begin{aligned}
\langle\ln \alpha\rangle & =-R e_{+}^{1 / 3} \\
\left\langle\ln ^{2} \alpha\right\rangle & =-\langle\ln \alpha\rangle
\end{aligned}
$$

where $R e_{+}=u_{*} h / \nu$.

The main properties of the stochastic process (14) are: (i) it providing a subgrid forcing that is correlated across the channel height; (ii) as the wall distance increases, it generates evolution of frequency from high to low mean values through two intermediate universal asymptotics: the earlier is log-normal distribution, while the latter is power law, with high probability of zero-frequency and stretched tail of high-frequency. This stretched tail is determined by $R e_{+}$, and induces rare events of strong subgrid forcing in the outer-flow, thereby mimicking bursting effects.

The starting condition (first grid cell, $\tau=0$ ) is given as follows. First, the mean value of the frequency at the wall is introduced as $f_{+}=u_{*} / \lambda$, where $\lambda$ is a Taylorlike scale. It is estimated by Kolmogorov's scaling in terms of wall parameters. The Reynolds number, based on friction velocity, is: $R e_{+}=u_{*} h / \nu=h / y_{0} \approx R e_{h}^{3 / 4}$, where $y_{0}$ is the thickness of the viscous layer, and $R e_{h}$ is the Reynolds number based on the center-line velocity. One then yields: $\lambda \approx h R e_{h}^{-1 / 2} \approx h R e_{+}^{-2 / 3}$. Second, the starting value for the random path (14) is sampled from the stationary log-normal distribution of $f / f_{+}$.

The probability density function (PDF) of the frequency $f$ has been computed for different wall distances by means of DNS in a channel flow with $R e_{+}=590$. Details about this simulation are given in Table 1. First, the norm of the acceleration was computed and then $f$ was estimated by using Equation (10). As it can be seen from Fig. 1, surprisingly, the model (Equations 14 and 15) matches well the results of DNS. In agreement with DNS data, the stochastic process provides a broad frequency distribution in the wall region and relaxes toward low frequencies when distance from the wall increases.

\subsection{Model for the orientation of the acceleration vector}

We assumed that with increasing distance from the wall, the orientation of subgrid acceleration relaxes stochastically from the streamwise-spanwise alignment (with 
random azimuth) to full isotropy.

First, this assumption was assessed by DNS performed in this paper. The orientation vector $e_{i}$ in Equation (9) is determined by longitude $\phi$ and latitude $\theta$ :

$$
e_{i}=\frac{\hat{a}_{i}^{\prime}}{|a|}=\left\{\begin{array}{l}
e_{x}=\cos (\theta) \cos (\phi) \\
e_{y}=\sin (\theta) \\
e_{z}=\cos (\theta) \sin (\phi)
\end{array}\right.
$$

where $-\pi \leq \phi \leq \pi$ characterizes orientation in the streamwise-spanwise $(x, z)$ plane, and $-\pi / 2 \leq \theta \leq \pi / 2$ defines orientation relatively to the normal-to-wall direction $(\theta=0$ and $\theta= \pm \pi / 2$ will correspond to accelerations, which are parallel to the wall and normal to the wall, respectively). The schematic representation is given in Fig. 2. If $\phi$ and $\theta$ are random, their PDF's corresponding to full isotropy, have the following forms $P_{\text {isotropic }}(\phi)=1 / 2 \pi$ and $P_{\text {isotropic }}(\theta)=|\cos (\theta)| / 2$, respectively. The mean value for both distributions is zero, and computation of variance yields:

$$
\begin{gathered}
<\phi^{2}>_{\text {isotropic }}=\int_{-\pi}^{\pi} \phi^{2} P_{\text {isotropic }}(\phi) d \phi=\frac{\pi^{2}}{3} \\
<\theta^{2}>_{\text {isotropic }}=\int_{-\pi / 2}^{\pi / 2} \theta^{2} P_{\text {isotropic }}(\theta) d \theta=\frac{\pi^{2}}{4}-2
\end{gathered}
$$

By DNS at $R e_{+}=180,590$ and 1000, one can obtain the statistics of $\phi$ and $\theta$ (see Section 3, and especially, Table 1 for details about the resolution used in the simulation). Then, $<\phi^{2}>$ and $<\theta^{2}>$ can be computed and compared with (17) and (18), respectively. To this end, large-scale acceleration obtained by our DNS has been removed by using sharp spectral filtering in homogeneous directions and Gaussian filtering in the normal to the wall direction. In such filtering operation, the mean of these two angles is negligibly small. The results of computations are given in Fig. 3 for different distances from the wall. It can be seen that the variance of $\phi$ (Fig. 3a) is close to the variance of the isotropic distribution (Equation 17) for almost the entire range of $y^{+}$, and for all three Reynolds numbers. As to variance of $\theta$ (Fig. 3b), it is negligibly small at the wall, and then it grows almost linearly with the distance from the wall up to $y^{+} \approx 30 \sim 50$, after which it tends towards a constant value. This value is relatively close to the variance of the isotropic distribution of $\theta$ (Equation 18). From this observation, we conclude that the subgrid acceleration vector relaxes towards isotropy, reaching at a high Reynolds number a nearly isotropic distribution at about $y^{+} \approx 50$.

In order to reflect this tendency towards isotropy, the stochastic model for the orientation (16) is constructed as a Brownian random walk on the surface of a sphere of unity radius. This random walk takes place with increasing distance from the wall. The evolution of the unit vector $e_{i}$ is defined by the following stochastic process:

$$
\left\{\begin{array}{l}
\gamma=2 D d W \\
0 \leq \beta<2 \pi
\end{array}\right.
$$

where $\gamma$ is the path length between two successive positions on the sphere, and $\beta$ is the initial direction from point 1 to point 2 (see Fig. 2 ). $\beta$ is chosen randomly from the uniform distribution. $D$ is a diffusion coefficient, and $d W$ is the standard 
Wiener process defined by: $\langle d W\rangle=0,\left\langle d W^{<}\right\rangle=2 d y^{+}$, with $d y^{+}$representing the cell size (in wall units) in the wall normal direction. The rules of this random walk are given by geodesic calculus:

$$
\left\{\begin{array}{l}
\theta_{k+1}=\sin ^{-1}\left(\sin \theta_{k} \cos \gamma+\cos \theta_{k} \sin \gamma \cos \beta\right) \\
\phi_{k+1}=\phi_{k}+\arg (\xi) \\
\Re(\xi)=\sin \beta \sin \gamma \cos \theta_{k} \\
\Im(\xi)=\cos \gamma-\sin \theta_{k} \sin \theta_{k+1}
\end{array}\right.
$$

where $\theta_{k}$ and $\phi_{k}$ are two angles corresponding to the node $k$, and $\xi$ is a complex number, with real and imaginary part $\Re(\xi)$ and $\Im(\xi)$, respectively.

With increasing distance from the wall $\left(y^{+}\right)$, the diffusive equilibrium is attained as a final state, which corresponds to the isotropic PDF's:

$$
\left\{\begin{aligned}
P_{\theta}\left(\theta, y^{+} \rightarrow \infty\right) & \rightarrow P_{\text {isotropic }}(\theta) \\
P_{\phi}\left(\phi, y^{+} \rightarrow \infty\right) & \rightarrow P_{\text {isotropic }}(\phi)
\end{aligned}\right.
$$

where, at the distance from the wall $y^{+}, P_{\theta}\left(\theta, y^{+}\right)$and $P_{\phi}\left(\phi, y^{+}\right)$are the PDF's of $\theta$ and $\phi$, respectively. An example of one realization of such a Brownian motion on a sphere is given in Fig. 4.

Correspondingly to DNS, the process starts on the wall $\left(y^{+}=0\right)$ with:

$$
\left\{\begin{array}{c}
P_{\theta}\left(\theta, y^{+}=0\right)=\delta(\theta) \\
P_{\phi}\left(\phi, y^{+}=0\right)=P_{\text {isotropic }}(\phi)
\end{array}\right.
$$

where $\delta$ is the Dirac distribution. This implies that the subgrid scale acceleration at $y^{+}=0$ is taken to be parallel to the wall with random azimuth.

In our case, the diffusion coefficient $D$ controls the relaxation rate towards isotropy. A linear regression of DNS data (Fig. 3b for $y^{+}<20$ ), yields that $D=0.01$. Fig. 5 shows statistics of $\theta$ computed from the model proposed here (Equations 19, 20 and 22, and $D=0.01$ ) and from our DNS. It can be seen that the model matches well the DNS data. For $\phi$, with the prescribed wall condition (22), the model provides exactly $<\phi^{2}>=<\phi^{2}>_{\text {isotropic }}$.

\section{Characteristics of the numerical simulations}

For assessment of the SGS acceleration model, we ran simulations of a pressure driven turbulent channel flow. A pseudo-spectral computational code is used. The code uses the spectral approximation (Fourier Chebyshev) and a variational projection method on a divergence free space following the original idea of Moser [45]. The time integration uses the explicit Adam-Basforth algorithm for non-linear terms, and the semi-implicit algorithm for diffusion terms. A rotational form is used for convective terms in order to ensure energy conservation. Periodic boundary conditions were applied along the streamwise $(x)$ and the spanwise $(z)$ directions, whereas the no-slip condition is imposed on the walls. The computer code was developed in the scientific group of M. Buffat [46].

A posteriori tests have been done for three Reynolds numbers: $R e_{+}=590,1000$ et 2000. Results of LES-SSAM approach were compared with the standard LES

and the DNS given in the literature $[47,48]$ and also with the DNS performed specifically in the present work. The conditions for those three approaches were exactly the same, including the same computational mesh for LES and LES-SSAM. The parameters taken for these simulations are summarized in Table 1. 
ıade 1. Summery or parameters used ror numerical simulations.

\begin{tabular}{lcccccccc}
\hline Name & $R e_{+}$ & $R e_{c}$ & $N_{x} \times N_{y} \times N_{z}$ & $L_{x} \times L_{y} \times L_{z}$ & $\Delta x^{+} \times \Delta y^{+} \times \Delta z^{+}$ & $d t^{+\mathrm{a}}$ & $C_{s}$ & $A / h$ \\
\hline DNS & 180 & 3280 & $192 \times 193 \times 192$ & $3 \pi h \times 2 h \times \frac{4}{3} \pi h$ & $9.0 \times(0.02 \sim 3.0) \times 4.0$ & 0.030 & - \\
\hline DNS & 587 & 12490 & $384 \times 257 \times 384$ & $\frac{3}{2} \pi h \times 2 h \times \frac{3}{4} \pi h$ & $7.2 \times(0.04 \sim 7.2) \times 3.6$ & 0.033 & - \\
DNS $^{b}$ & 587 & 12547 & $384 \times 257 \times 384$ & $2 \pi h \times 2 h \times \pi h$ & $9.7 \times(0.04 \sim 7.2) \times 4.8$ & - & - \\
LES & 587 & 14160 & $64 \times 65 \times 64$ & $3 \pi h \times 2 h \times \pi h$ & $87 \times(0.71 \sim 29) \times 29$ & 0.1 & 0.16 & 0.015 \\
LES-SSAM & 587 & 12760 & $64 \times 65 \times 64$ & $3 \pi h \times 2 h \times \pi h$ & $87 \times(0.71 \sim 29) \times 29$ & 0.1 & 0.16 & 0.015 \\
\hline DNS & 1000 & 22250 & $512 \times 385 \times 512$ & $\frac{4}{3} \pi h \times 2 h \times \frac{2}{3} \pi h$ & $8.2 \times(0.03 \sim 8.3) \times 4.1$ & 0.034 & - & - \\
DNS & 934 & 20960 & $3072 \times 385 \times 2304$ & $8 \pi h \times 2 h \times 3 \pi h$ & $7.6 \times(0.06 \sim 7.6) \times 3.8$ & - & - \\
LES & 1000 & 25430 & $96 \times 97 \times 96$ & $3 \pi h \times 2 h \times \pi h$ & $99 \times(0.53 \sim 33) \times 33$ & 0.1 & 0.16 & 0.009 \\
LES-SSAM & 1000 & 23380 & $96 \times 97 \times 96$ & $3 \pi h \times 2 h \times \pi h$ & $99 \times(0.53 \sim 33) \times 33$ & 0.1 & 0.16 & 0.009 \\
LES & 1000 & 25500 & $64 \times 65 \times 64$ & $3 \pi h \times 2 h \times \pi h$ & $147 \times(1.2 \sim 49) \times 49$ & 0.1 & 0.2 & 0.015 \\
LES-SSAM & 1000 & 23700 & $64 \times 65 \times 64$ & $3 \pi h \times 2 h \times \pi h$ & $147 \times(1.2 \sim 49) \times 49$ & 0.1 & 0.2 & 0.015 \\
\hline DNS & 2003 & 48680 & $6144 \times 633 \times 4608$ & $8 \pi h \times 2 h \times 3 \pi h$ & $8.2 \times 8.9 \times 4.1$ & - & - \\
LES & 2000 & 49350 & $128 \times 129 \times 128$ & $3 \pi h \times 2 h \times \pi h$ & $147 \times(0.60 \sim 49) \times 49$ & 0.1 & 0.16 & 0.006 \\
LES-SSAM & 2000 & 48950 & $128 \times 129 \times 128$ & $3 \pi h \times 2 h \times \pi h$ & $147 \times(0.60 \sim 49) \times 49$ & 0.1 & 0.16 & 0.006 \\
LES & 2000 & 52640 & $64 \times 65 \times 64$ & $3 \pi h \times 2 h \times \pi h$ & $295 \times(2.4 \sim 98) \times 98$ & 0.1 & 0.2 & 0.015 \\
LES-SSAM & 2000 & 49050 & $64 \times 65 \times 64$ & $3 \pi h \times 2 h \times \pi h$ & $295 \times(2.4 \sim 98) \times 98$ & 0.1 & 0.2 & 0.015 \\
\hline
\end{tabular}

a $d t^{+}=d t / t_{*}$ is the time step of the simulation; $t_{*}=\nu / u_{*}^{2}$ is a viscous time of the order of the Kolmogorov time scale at the wall.

b Moser et al. (1999) [47]

c Hoyas and Jiménez (2008) [48]

For LES and LES-SSAM simulations, the classical Smagorinsky model is used with wall damping function for the turbulent viscosity [4]:

$$
\begin{aligned}
\nu_{\text {turb }} & =\left(C_{s} \Delta f_{V D}\right)^{2}|S| \\
|S| & =\left(2 S_{i j} S_{i j}\right)^{1 / 2} \\
f_{V D} & =1-e^{-y / A}
\end{aligned}
$$

where $\mathrm{A}$ is specified below. Here $C_{s}$ is the Smagorinsky constant, $\Delta=(\Delta x \times \Delta y \times$ $\Delta z)^{1 / 3}$ is the characteristic grid size, $S_{i j}=\frac{1}{2}\left(\frac{\partial \bar{u}_{i}}{\partial x_{j}}+\frac{\partial \bar{u}_{j}}{\partial x_{i}}\right)$ is the resolved strainrate tensor, $f_{V D}$ is the Van Driest function, $y$ is the distance from nearest wall, and $A$ is the constant controlling the damping function $f_{V D}$. As suggested by [49], for a detached-eddy simulation (DES), the turbulent length-scale in the wall-layer is defined as $\ell=\min (y, \Delta)$. In order to fulfill this suggestion, in the Van Driest damping function, we choose $A$ such that $\Delta \times f_{V D} \sim \min (y, \Delta)$.

It should be noted that in this code, the Reynolds number is given by choosing $\nu$ and the pressure gradient $-\frac{1}{\rho} \frac{\partial P_{t}}{\partial x}$. In order to attain the required Reynolds number we used the Dean's correlation [50]: $\nu=0.110 U_{c} h R e_{+}^{-1.1296}$ and $-\frac{1}{\rho} \frac{\partial P_{t}}{\partial x}=$ $R e_{+}^{2} \nu^{2} / h^{3}$, where $U_{c}$ is the center-line velocity. It can be noticed from Table 1 that the LES-SSAM matches better the DNS Reynolds number than the standard LES. Thereby, for a given set of parameters $\left(\nu\right.$ and $\left.-\frac{1}{\rho} \frac{\partial P_{t}}{\partial x}\right)$, both the center-line velocity and the mass flow rate are improved by LES-SSAM model.

\section{Results and discussion}

For the sake of simplicity, we will only present simulations for the $64 \times 65 \times 64$ grid, for all three Reynolds numbers. For finer resolutions the difference between LES-SSAM and standard LES is less pronounced, but still present. Fig. 6 shows evolution of the mean velocity across the channel. The LES-SSAM reproduces well 
the logarithmic law of the wall predicted by DNS, which is not the case with standard LES.

Fig. 7 shows also the improvement in the prediction of the velocity variance when LES-SSAM is used. This can be seen especially for the streamwise velocity compared to the DNS of [48]. For the other components, the profile is also better predicted by LES-SSAM than by the standard LES. It should be noted that the variance of the streamwise velocity at $R e_{+}=1000$ computed by our DNS deviates slightly from the DNS in [48]. This may be due to a smaller computational domain in comparison with [48] and a higher Reynolds number.

Fig. 8 illustrates the vertical profiles of the turbulent and viscous stresses, $\tau_{\text {turb }}=$ $-\rho\left\langle u^{\prime} v^{\prime}\right\rangle$ and $\tau_{v i s c}=-\rho \nu \frac{\partial\langle u\rangle}{\partial y}$, respectively. The results are presented as ratios $\tau_{\text {turb }} /\left(\tau_{\text {turb }}+\tau_{\text {visc }}\right)$ and $\tau_{\text {visc }} /\left(\tau_{\text {turb }}+\tau_{\text {visc }}\right)$. Once again, the advantage of the LESSSAM approach versus the classical LES is explicitly shown. Both the viscous and the turbulent stresses are better predicted by LES-SSAM.

Velocity spectra are illustrated in Fig. 9. For both Reynolds numbers and both $y^{+}$shown in the figure, it can be clearly seen that the high wave number part of the spectrum is much better resolved by LES-SSAM than by LES.

Fig. 10 represents the evolution of the longitudinal autocorrelation coefficient of the velocity components at $y^{+}=5$, representing the near-wall region. The improvement of the correlation length due to the better resolution of the small-scale part of the spectrum by LES-SSAM is clearly seen in this figure. Another observation is that for small wall distances, the streamwise longitudinal autocorrelation is more spatially correlated than the remaining two components, because of the alignment of streaky structures with the wall.

Fig. 11 illustrates the PDF of the spanwise acceleration. At three very different distances to the wall, the PDF obtained by the DNS displays stretched tails, as a manifestation of intermittency. The same evidence of intermittency is obtained by LES-SSAM, while in the case of the standard LES those tails are much less developed. This effect is also observed for the PDF of the other two components of the acceleration.

\section{Conclusion}

In numerical simulation of a high-Reynolds number channel flow at moderate resolution, the instantaneous acceleration has been split into filtered and subgrid parts. Then, both components were modeled, in the framework of LES combined with the stochastic subgrid acceleration model.

The aim of the new model for subgrid acceleration was to account for intermittency in the residual (unfiltered) wall-bounded flow. This model was constructed in accordance with DNS observations, reported in the literature, and DNS performed specifically in the present paper. The model is based on two independent stochastic processes: one for the norm of the subgrid acceleration, the other for its orientation.

The norm is given by a characteristic velocity increment, in terms of wallparameters, multiplied by stochastic frequency. The stochastic process, constructed in lines of statistical universalities in fragmentation under the scaling symmetry, evolves with distance from the wall towards outer flow. To this end the nondimensional evolution parameter was introduced, which increases from zero to infinity, as the distance to the wall increases from the wall to the channel half- 
width. 'I'he multiplicative stochastic process provides correlation of the subgrid forcing across the channel. With increasing wall distance and consequently, decreasing mean frequency, this process has two intermediate universal asymptotics. The earlier is the log-normal distribution, while the later is power distribution. The power law has a high peak of probability for zero-frequency and a stretched tail for high-frequencies, which depends on the Reynolds number. Sampling from that stretched tail induces rare events of strong subgrid forcing in the outer-flow, mimicking thereby bursting events.

It was assumed that orientation of the subgrid acceleration is controlled by orientation of vortices, which become randomly distributed, if distance from the wall increases. In order to fulfill this tendency towards full isotropy, the stochastic model for subgrid acceleration orientation is based on a Brownian random walk on the surface of a sphere of unity radius. Starting close to the wall, from the direction in the streamwise-spanwise plane with random azimuth, the random walk takes place with increasing distance from the wall, and tends to the diffusive equilibrium as a final state, which corresponds to isotropic PDF's for the two geodesic angles, longitude and latitude. The diffusion coefficient was computed by DNS and found to be almost independent of the Reynolds number.

In the case of turbulent channel flow, the results of the LES-SSAM approach were compared with DNS data $\left(R e_{+}=590,1000\right.$ and 2000) and with standard LES. The LES-SSAM model was proven successful in predictions of DNS results: (i) resolution of the small-scale part of the energy spectrum and simulation of spatial correlations are close to DNS; (ii) stretched tails in the PDF of acceleration are predicted in agreement with DNS; (iii) computation of mean and of variance of the velocity components, as well as of turbulent and viscous stresses are explicitly improved. Along with fundamental interest, the LES-SSAM approach has a practical relevance, when significant physics take place on subgrid scales. In such conditions, simulations at a high Reynolds number warrant advanced SGS models that account for intermittency effects on small spatial scales. Examples include scalar mixing and turbulent combustion, dispersion, vaporization and combustion in twophase flows. This requires the further development of this approach with many associated questions, concerning complex geometry, correlation between norm and orientation of the subgrid acceleration, time-correlations, etc. However, the approach given in [14], and in the present paper is very simple, and easily realizable for any LES code without supplementary CPU requirement.

\section{Acknowledgments}

M. Buffat is acknowledged for the development of the computational code. The authors express their gratitude to F. Laadhari who kindly provided his initial fields for the DNS. It is a pleasure to thank V. Sabel'nikov, P. Moin and J. Jiménez for discussions on this subject with many benefits for us. This work was granted access to the HPC resources of CINES under the allocation 2009-c200902560 made by GENCI (Grand Equipement National de Calcul Intensif)

\section{References}

[1] P. Moin and J. Kim. Numerical investigation of turbulent channel flow. Journal of Fluid Mechanics, 118:341-377, 1982. 
[2] C. Meneveau and J. Katz. Scale-invariance and turbulence models for large-eddy simulation. Annual Reviews of Fluid Mechanics, 32:1-32, 2000.

[3] U. Piomelli and E. Balaras. Wall-layer models for large-eddy simulations. Annual Reviews of Fluid Mechanics, 34:349, 2002.

[4] P. Sagaut. Large Eddy Simulation for Incompressible Flows: An introduction. Springer Verlag, second ed edition, 2002.

[5] C. Pantano, D.I. Pullin, P.E. Dimotakis, and G. Matheou. Les approach for high reynolds number wall-bounded flows with application to turbulent channel flow. Journal of Computational Physics, 227(21):9271 - 9291, 2008. Special Issue Celebrating Tony Leonard's 70th Birthday.

[6] P. R. Spalart, S. Deck, M. L. Shur, K. D. Squires, M. KH. Strelets, and A. Travin. A new version of detached-eddy simulation, resistant to ambiguous grid densities. Theoretical and Computational Fluid Dynamics, 20:181-195, 2006.

[7] J. A. Domaradzki and N. A. Adams. Direct modelling of subgrid scales of turbulence in large eddy simulations. Journal of Turbulence, 3:0-24, 2002.

[8] N. Park and K. Mahesh. A velocity-estimation subgrid model constrained by subgrid scale dissipation. Journal of Computational Physics, 227:4190-4206, 2008.

[9] P. S. Westbury, D. C. Dunn, and J. F. Morrison. Analysis of a stochastic backscatter model for the large-eddy simulation of wall-bonded flow. European Journal of Mechanics B, 23:737-758, 2004.

[10] R. C. Schmidt, A. R. Kerstein, and S. Wunsch. Near-wall LES closure based on one-dimensional turbulence modelling. Journal of Computational Physics, 186:317-355, 2003.

[11] A. R. Kerstein. One-dimensional turbulence: model formulation and application to homogeneous turbulence, shear flows, and buoyant stratified flows. Journal of Fluid Mechanics, 392:277-334, 1999.

[12] A. R. Kerstein. One-dimensional turbulence: A new approach to high-fidelity subgrid closure of turbulent flow simulations. Computer Physics Communications, 148:1-16, 2002.

[13] K. A. Kemenov and S. Menon. Explicit small-scale velocity simulation for high-Re turbulent flows. Part II: Non-homogeneous flows. Journal of Computational Physics, 222:673-701, 2007.

[14] V. Sabel'nikov, A. Chtab, and M. Gorokhovski. The coupled LES - sub-grid stochastic acceleration model (LES-SSAM) of a high Reynolds number flows. In Advances in Turbulence XI, volume 117, pages 209-211, 11th EUROMECH European Turbulence Conference, June 25-28, 2007, Porto, Portugal, 2007. Springer Proceedings in Physics.

[15] C. Lee, K. Yeo, and J.-I. Choi. Intermittent nature of acceleration in near-wall turbulence. Physical Review Letters, 92(14), 2004.

[16] N. Mordant, E. Lévêque, and J.-F. Pinton. Experimental and numerical study of the Lagrangian dynamics of high Reynolds turbulence. New Journal of Physics, 6:116, 2004.

[17] K. Voth, G. A.and Satyanarayan and Bodenschatz E. Lagrangian acceleration measurements at large reynolds numbers. Physics of Fluids, 10:2268, 1998.

[18] G.A. Voth, A. La Porta, A.M. Grawford, J. Alexander, and Bodenschatz E. Measurements of particle accelerations in fully developed turbulence. Journal of Fluid Mechanics, 469:121, 2002.

[19] J. Jiménez. Recent developments on wall-bounded turbulence. Rev. R. Acad. Cien. Serie A. Mat., 101(2):187-203, 2007.

[20] J. Kim. On the structure of wall-bonded flows. Physics of Fluids, 26(8), 1983.

[21] J. M. Hamilton, J. Kim, and F. Waleffe. Regeneration mechanisms of ner-wall turbulence structures. Journal of Fluid Mechanics, 287:317-348, 1995.

[22] J. Jiménez and A. Pinelli. The autonomous cycle of near-wall turbulence. Journal of Fluid Mechanics, 389:335-359, 1999.

[23] J. Jiménez, J. C. Del Alamo, and O. Flores. The large-scale dynamics of near-wall turbulence. Journal of Fluid Mechanics, 505:179-199, 2004.

[24] C. R. Smith and S. P. Metzler. The characteristics of low-speed streaks in the near-wall region of a turbulent boundary layer. Journal of Fluid Mechanics, 129:27-54, 1983.

$[25]$ J. D. Swearingen and R. F. Blackwelder. The growth and breakdown of streamwise vortices in the presence of a wall. Journal of Fluid Mechanics, 182:255-290, 1987.

[26] I. Marusic and G. J. Kunkel. Streamwise turbulence intensity formulation for flat-plate boundary layers. Physics of Fluids, 15(8), 2003.

[27] N. Hutchins and I. Marusic. Evidence of very long meandering features in the logarithmic region of turbulent boundary layers. Journal of Fluid Mechanics, 579:1-28, 2007.

[28] J. Jiménez. The largest structures in turbulent wall flows. In CTR Annual Research Briefs, pages 943-945, 1998.

[29] J. C. Del Alamo and J. Jiménez. Linear energy amplification in turbulent channels. Journal of Fluid Mechanics, 559:205-213, 2006.

[30] J. Jiménez and S. Hoyas. Turbulent fluctuations above the buffer layer of wall-bounded flows. Journal of Fluid Mechanics, 611:215-236, 2008.

[31] J. C. Del Alamo, J. Jiménez, P. Zandonade, and R. D. Moser. Scaling of the energy spectra of turbulent channels. Journal of Fluid Mechanics, 500:135-144, 2004.

[32] S. Toh and T. Itano. Interaction between a large-scale structure and near-wall structures in channel flow. Journal of Fluid Mechanics, 524:249-262, 2005.

[33] J. C. Del Alamo, J. Jiménez, P. Zandonade, and R. D. Moser. Self-similar vortex clusters in the turbulent logarithmic region. Journal of Fluid Mechanics, 561:329-358, 2006.

[34] J. Jiménez, G. Kawahara, M.P. Simens, M. Nagata, and M. Shiba. Characterization of near-wall turbulence in terms of equilibrium and "bursting" solutions. Physics of Fluids, 17:015105, 2005.

[35] C. Härtel, L. Kleiser, F. Unger, and R. Friedrich. Subgrid-scale energy transfert in the near-wall region of turbulent flows. Physics of Fluids, 6(9):3130-3143, 1994.

[36] A. N. Kolmogorov. A refinement of previous hypotheses concerning the local structure of turbulence in a viscous incompressible fluid at high Reynolds number. Journal of Fluid Mechanics, 13:82-85, 1962.

[37] A. M. Oboukhov. Some specific features of atmospheric tubulence. Journal of Fluid Mechanics, 
13(01):77-81, 1962

[38] A. S. Monin and A. M. Yaglom. Statistical Fluid Mechanics: Mechanics of Turbulence. MIT Press, 1981.

[39] S. B. Pope and Y. L. Chen. The velocity-dissipation probability density function model for turbulent flows. Physics of Fluids, 2(8):1437-1449, 1990.

[40] A. G. Lamorgese, S. B. Pope, P. K. Yeung, and B. L. Sawford. A conditionally cubic-Gaussian stochastic Lagrangian model for acceleration in isotropic turbulence. Journal of Fluid Mechanics, $582: 243-448,2007$.

[41] A. Arneodo, S. Manneville, and J.F. Muzy. Towards log-normal statistics in high Reynolds number turbulence. European Physical Journal B, 1(1):129, 1998.

[42] V. Sabel'nikov, M. Gorokhovski, and N. Baricault. The extended iem mixing model in the framework of the composition pdf approach: applications to diesel spray combustion. Combustion Theory and Modelling, 10(1):155-169, 2006.

[43] M. A. Gorokhovski and V. L. Saveliev. Statistical universalities in fragmentation under scaling symmetry with a constant frequency of fragmentation. Journal of Physics D: Applied Physics, 41:085405, 2008

[44] A. N. Kolmogorov. On the log-normal distribution of particles sizes during break-up process. Dokl. Akad. Nauk SSSR, 31(99), 1941.

[45] R. D. Moser, P. Moin, and A. Leonard. A spectral numerical method for the Navier-Stokes equations with applications to Taylor-Couette flow. Journal of Computational Physics, 52:524-544, 1983.

[46] H. Pascal. Etude d'une turbulence compressée et ou cisaillée entre deux plans parallèles: comparaison entre approche statistique et simulation des équations de Navier-Stokes instantanées. PhD thesis, Ecole centrale de Lyon, 1996. Buffat M. (Directeur de thèse).

[47] R. D. Moser, J. Kim, and N. N. Mansour. Direct numerical simulation of turbulent channel flow up to $R e_{\tau}=590$. Physics of Fluids, 11(4):943-945, 1999.

[48] S. Hoyas and J. Jiménez. Reynolds number effects on the reynolds-stress budgets in turbulent channels. Physics of Fluids, 20(10):101511, 2008.

[49] M. L. Shur, P. R. Spalart, M. Kh. Strelets, and A. K. Travin. A hybrid RANS-LES approach with delayed-DES and wall-modelled LES capabilities. International Journal of Heat and Fluid Flow, 29(6):1638-1649, 2008.

[50] R. B. Dean. Reynolds Number dependance on skin Friction and other bulk flow variables in twodimensional rectangular duct flow. Journal of Fluids Engineering, 100:216-223, 1978. 


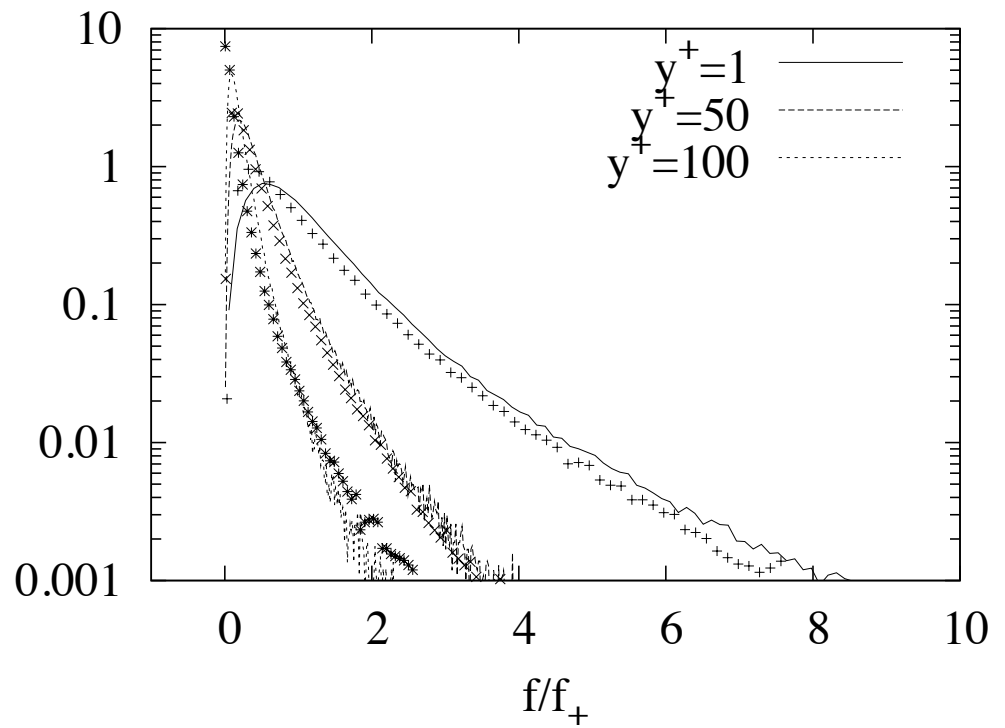

Figure 1. Distribution of $f / f_{+}$from SSAM (cross) and comparison with DNS (line) at $R e_{+}=590$, for several distances from the wall.

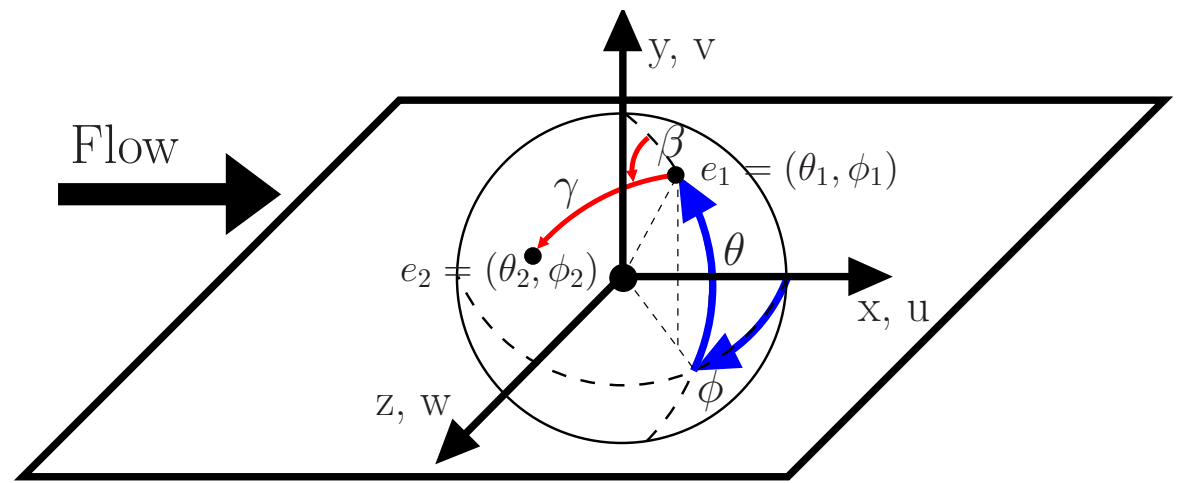

Figure 2. Coordinate system, and definition of the angles $\phi, \theta, \gamma$ and $\beta$. 


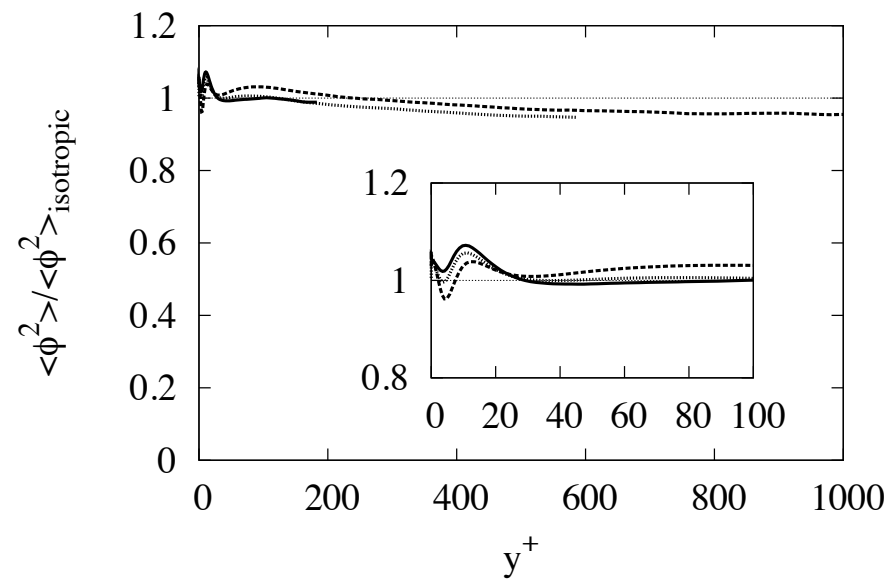

(a)

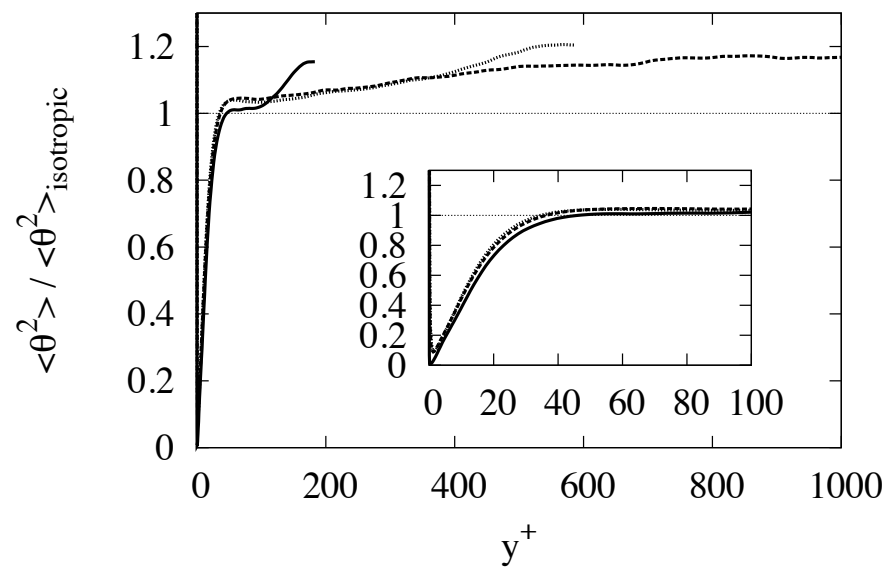

(b)

Figure 3. Variance of the angles $\phi$ (a) and $\theta$ (b) for the orientation of the acceleration at small scale obtained from DNS, for $R e_{+}=180$ (full line) $R e_{+}=590$ (dotted line) and $R e_{+}=1000$ (dashed line). The variances are normalized by the value of the variance for an isotropic distribution of the orientation vector (Equations 17 and 18). Insert: zoom near the wall. 


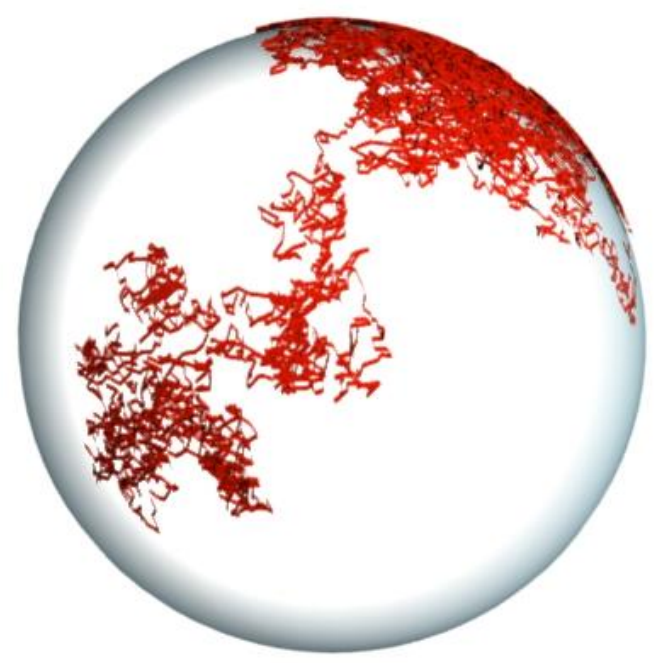

Figure 4. Realization of the random walk on the sphere given by Equations 19 and 20.

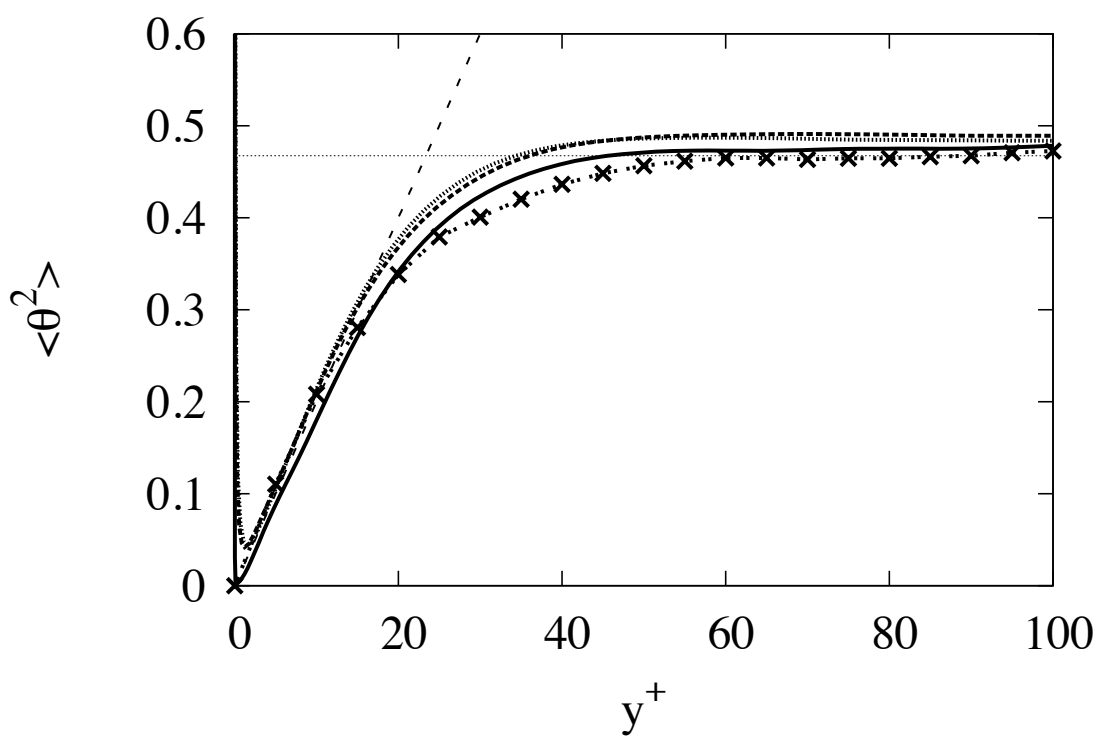

Figure 5. Evolution of the variance of $\theta$. Comparison between DNS for $R e_{+}=180$ (full line) $R e_{+}=590$ (dotted line) and $R e_{+}=1000$ (dashed line) and the stochastic model defined by Equations 19, 20 and 22 (crosses). The straight line corresponds to the variance for an isotropic distribution. 


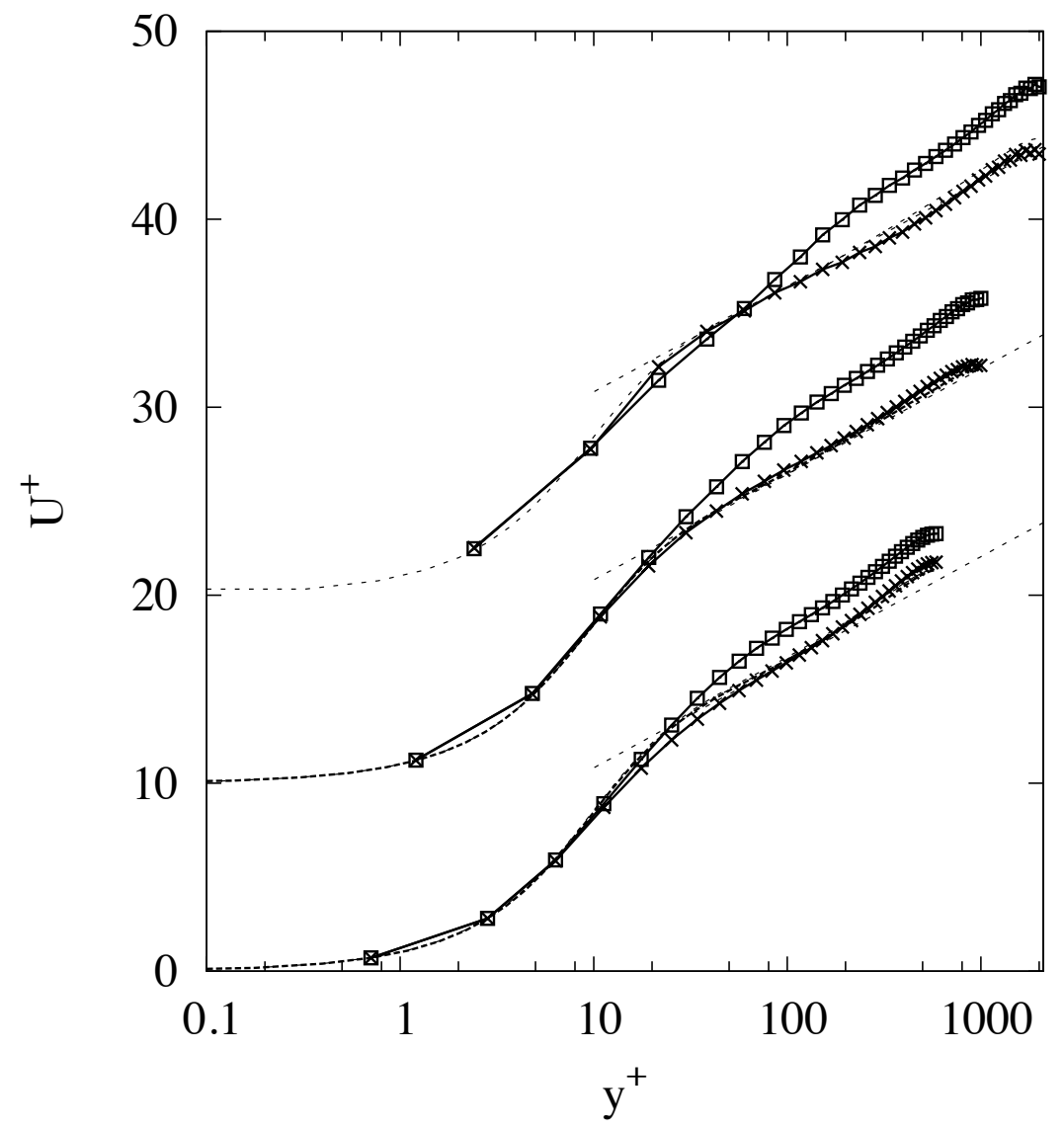

Figure 6. Streamwise mean velocity, for $R e_{+}=590, R e_{+}=1000$ and $R e_{+}=2000$ from bottom to top, respectively, shifted by 10 wall units upward. Square: LES; cross: LES-SSAM; dash: DNS (only for $R e_{+}=590$ and $R e_{+}=1000$ ); dots: DNS from [47] for $R e_{+}=590$ and from [48] for $R e_{+}=1000$ and $R e_{+}=2000$. Long dashed line: logarithmic law of the wall. 


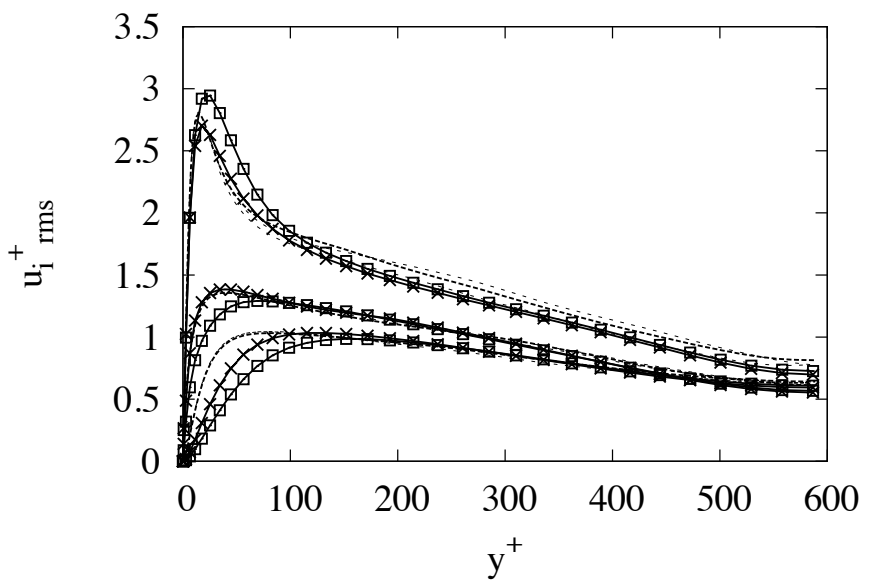

(a)

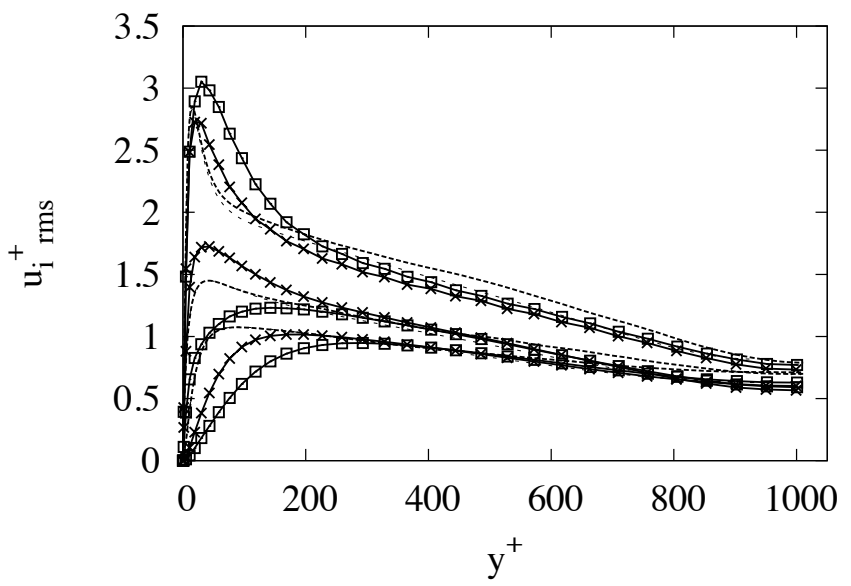

(b)

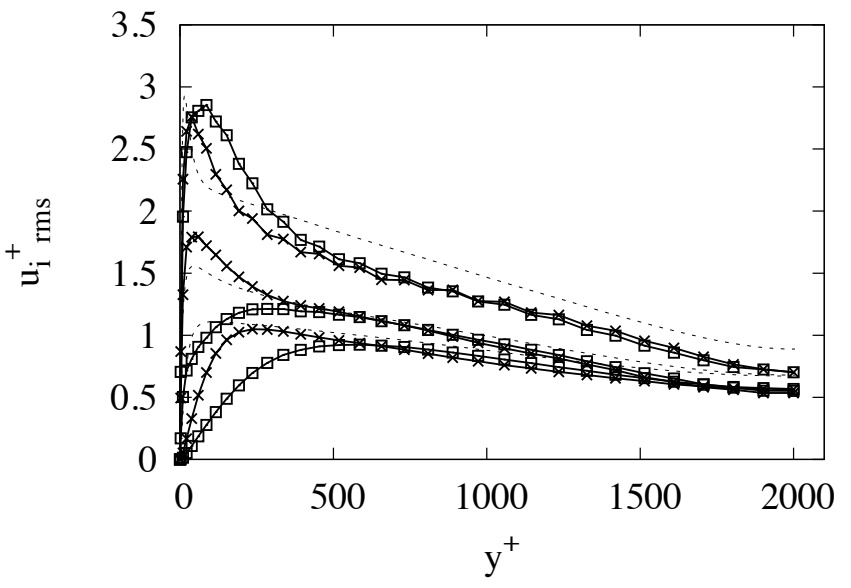

(c)

Figure 7. Standard deviation of streamwise $(u)$, spanwise $(w)$ and normal $(v)$ velocity (in wall unites), for $R e_{+}=590$ (a), $R e_{+}=1000$ (b) and $R e_{+}=$ 2000 (c), from top to bottom, respectively. Square: LES; cross: LES-SSAM; dash: DNS (only for $R e_{+}=590$ and $R e_{+}=1000$ ); dots: DNS from [47] for $R e_{+}=590$ and from [48] for $R e_{+}=1000$ and $R e_{+}=2000$ 


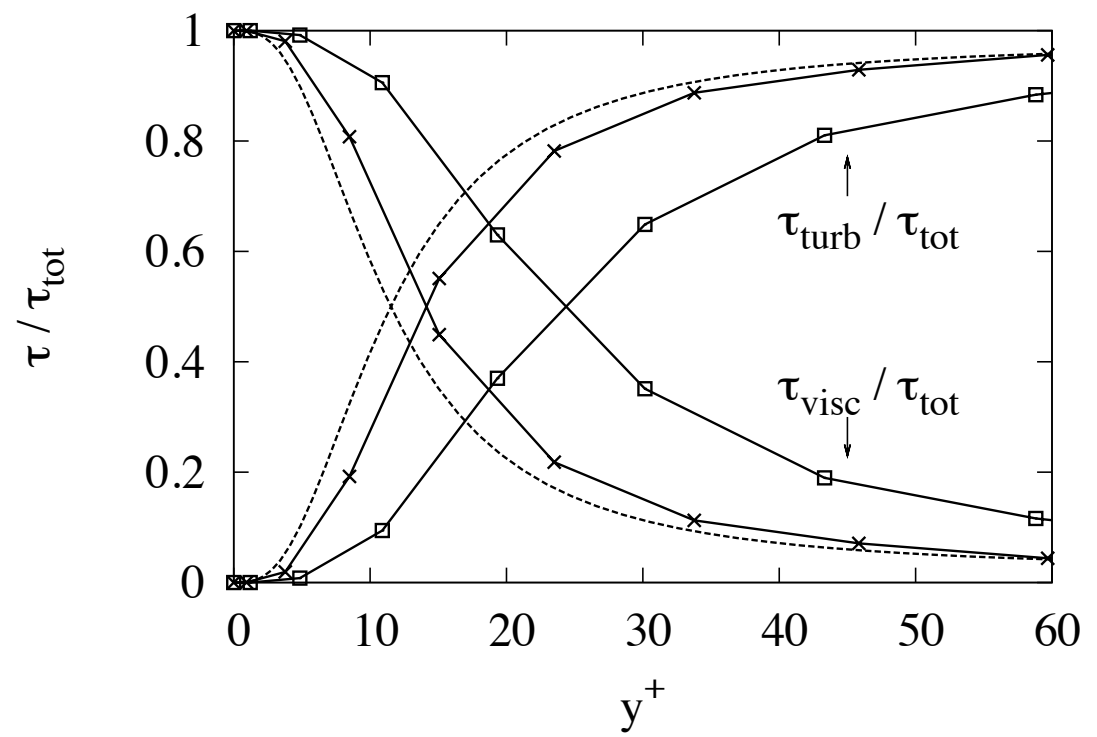

Figure 8. Ratios of turbulent $\tau_{\text {turb }}=-\rho\left\langle u^{\prime} v^{\prime}\right\rangle$ and viscous $\tau_{v i s c}=-\rho \nu\left\langle\frac{\partial u}{\partial y}\right\rangle$ stresses compared to the total one $\tau_{t o t}=\tau_{v i s c}+\tau_{\text {turb }}$. Re $+=1000$. Square: LES; cross: LES-SSAM; dash: DNS. 


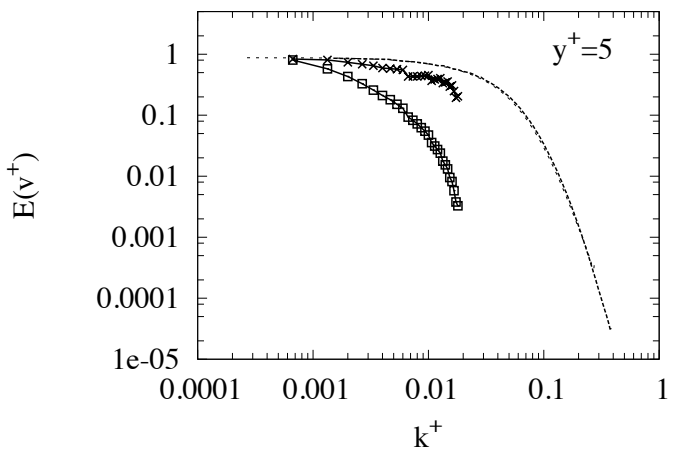

(a)

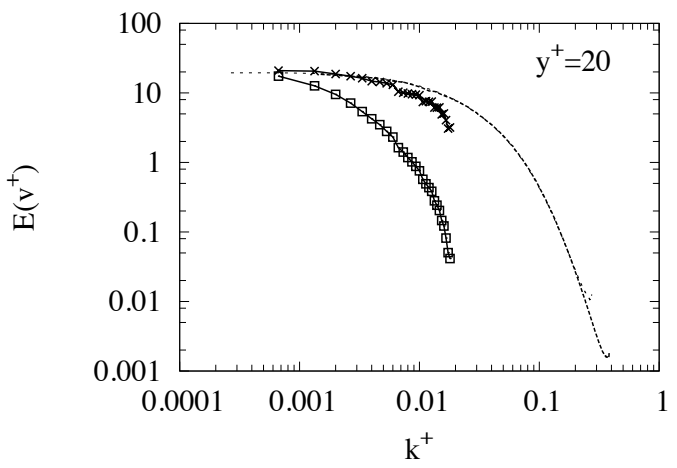

(b)

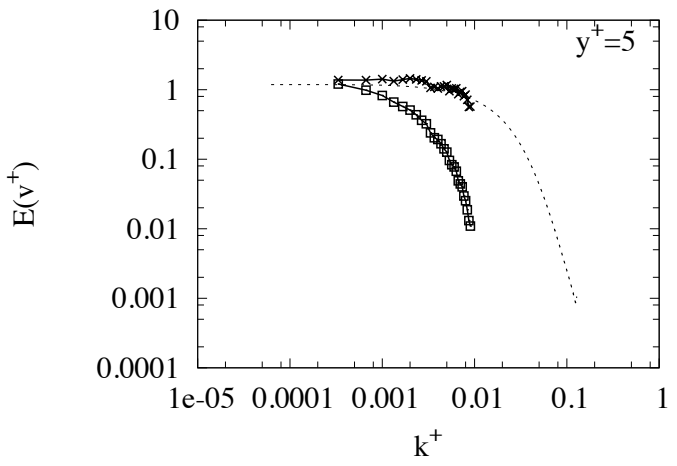

(c)

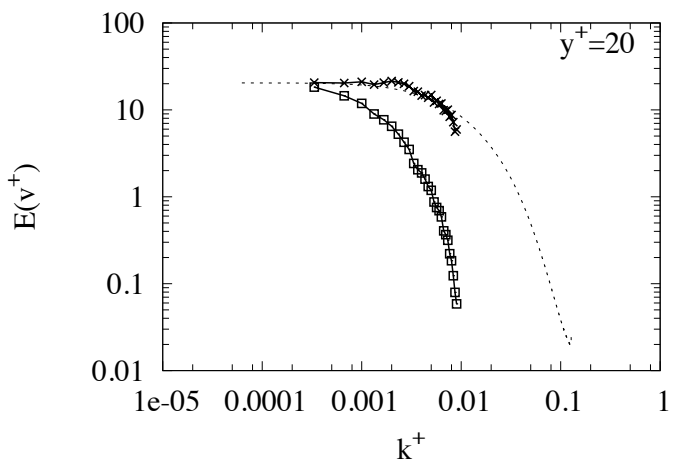

(d)

Figure 9. Normalized longitudinal one dimensional spectra of normal to wall velocity for two distances to the wall : $y^{+}=5$ and $y^{+}=20$, for $R e_{+}=1000$ (a and b) and $R e_{+}=2000$ (c and d). Square: LES; cross: LES-SSAM; dash: DNS dots: DNS from [48]. 


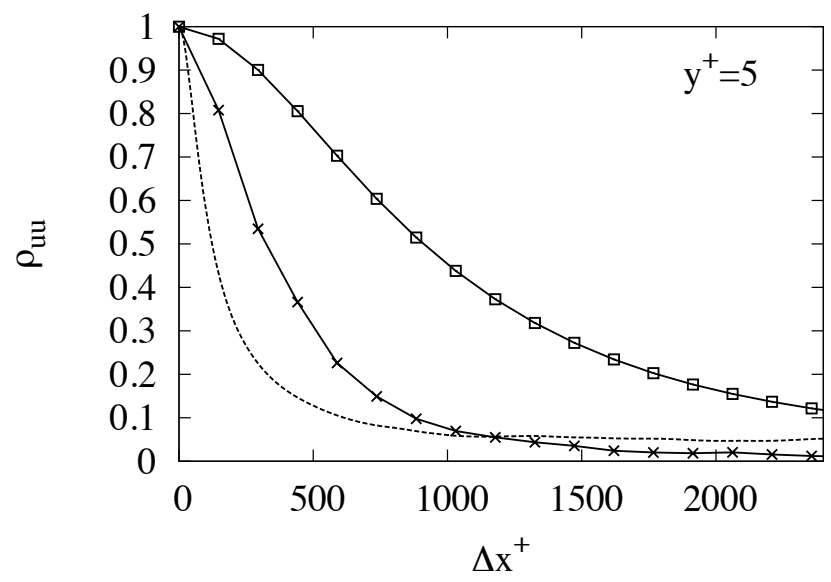

(a)

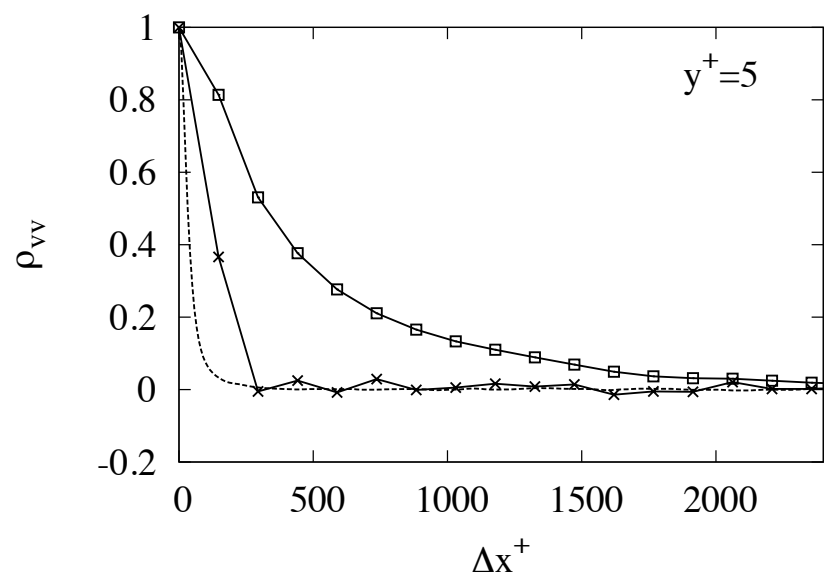

(b)

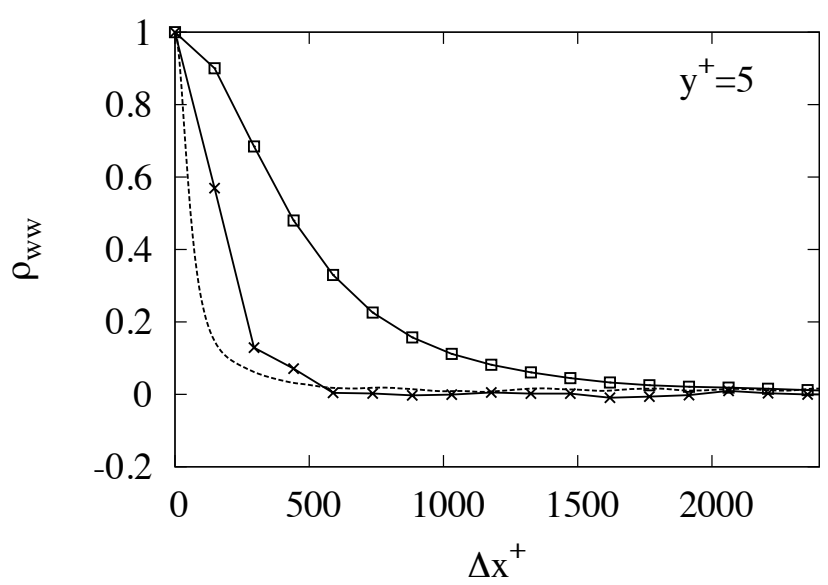

(c)

Figure 10. Longitudinal autocorrelation coefficient of streamwise (a), normal (b) and spanwise (c) velocity component, at $y^{+}=5, R e_{+}=1000$. Square: LES; cross: LES-SSAM; dash: DNS. 


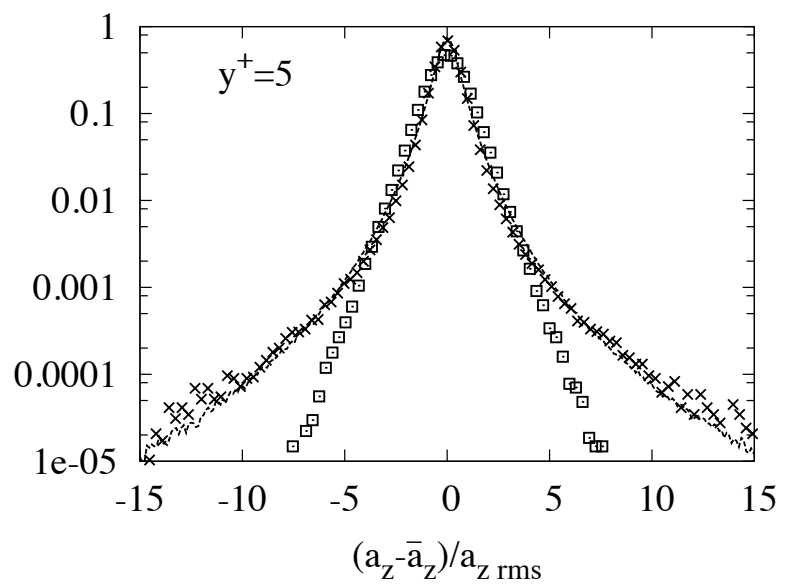

(a)

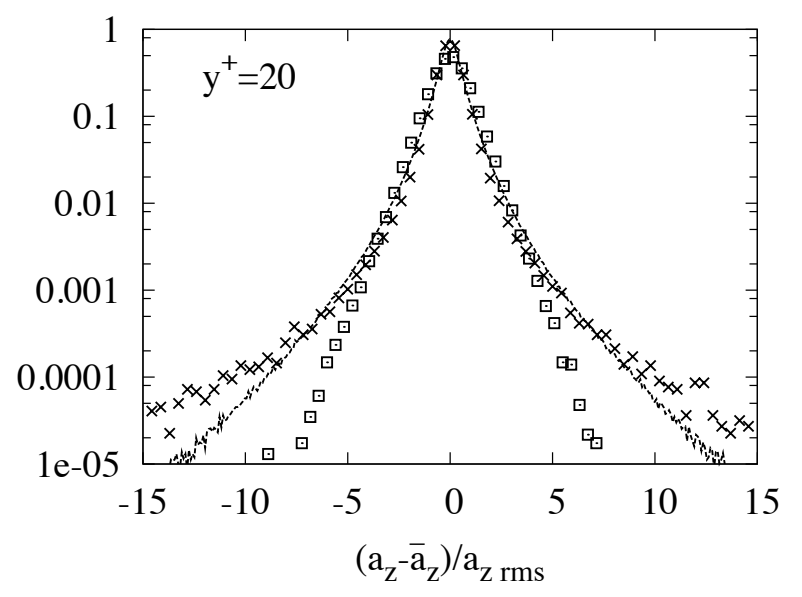

(b)

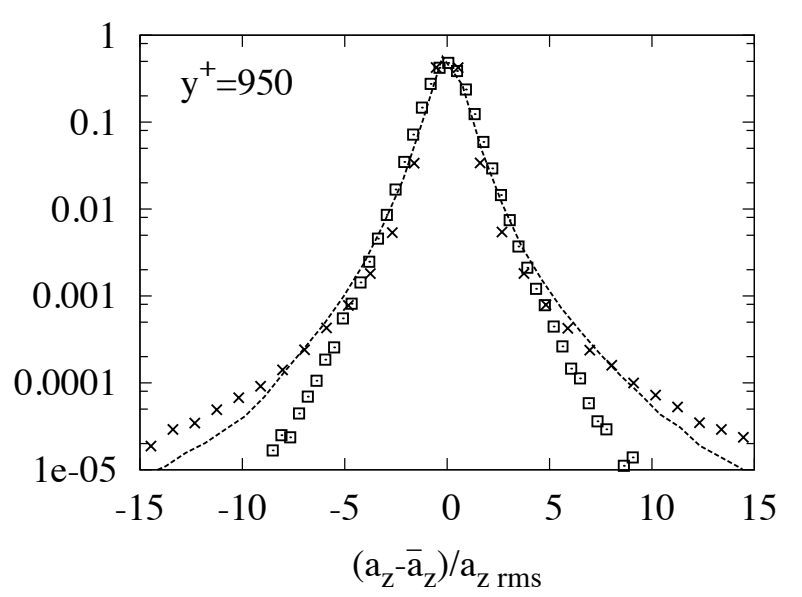

(c)

Figure 11. Distribution of spanwise acceleration at three distances from the wall: $y^{+}=5, y^{+}=20, y^{+}=950$. $R e_{+}=1000$. Square: LES; cross: LESSSAM; dash: DNS. 